\title{
Mechanical Efficiency of HMCVT under Steady-State Conditions
}

\author{
Guangqing Zhang, ${ }^{1}$ Hengtong Zhang $\mathbb{D}^{2}$ Yanyan Ge, ${ }^{2}$ Wei Qiu $\mathbb{D}^{2},{ }^{2}$ Maohua Xiao $\mathbb{D}^{2}$ \\ Xiaomei Xu, ${ }^{3}$ and Minghui Zhou ${ }^{4}$ \\ ${ }^{1}$ Xuzhou Carter Agricultural Equipment Co., Ltd., Xuzhou 221011, China \\ ${ }^{2}$ College of Engineering, Nanjing Agricultural University, Nanjing 210031, China \\ ${ }^{3}$ College of Automobile and Traffic Engineering, Nanjing Forestry University, Nanjing 210042, China \\ ${ }^{4}$ Weichai Power Hydraulic Transmission Research Institute, Weifang, 261001, China \\ Correspondence should be addressed to Wei Qiu; qiuwei@njau.edu.cn
}

Received 19 June 2021; Accepted 11 August 2021; Published 7 September 2021

Academic Editor: Jun Zhu

Copyright (c) 2021 Guangqing Zhang et al. This is an open access article distributed under the Creative Commons Attribution License, which permits unrestricted use, distribution, and reproduction in any medium, provided the original work is properly cited.

\begin{abstract}
Hydromechanical continuously variable transmission (HMCVT) technology has been widely used due to its advantages of ride comfort and fuel economy. The relatively uniform efficiency expression of HMCVT is obtained by studying torque and transmission ratios to reveal steady-state characteristics and predict the output torque. Mathematical models of torque ratios are derived by analyzing the HMCVT system power flow and calculating the equivalent meshing power of epicyclic gear train and efficiency for the hydraulic system. The relationship between mechanical system transmission and hydraulic system parameters is established using the torque ratios, and a mechanical system demanding surface is proposed. Two numerical examples of the HMCVT system with single and dual variable units are demonstrated to establish an effective and convenient method. The method is validated through a physical prototype TA1-02 test.
\end{abstract}

\section{Introduction}

An epicyclic gear train (EGT) with two DOFs is closed by a hydrostatic transmission system (HST), so that only one DOF exists overall [1]. The mechanical hydraulic components are organically combined to form a simple hydromechanical continuously variable transmission (HMCVT) $[2,3]$. When the input speed of HMCVT is stable, with displacement ratio $e$ of HST changing continuously in a certain range, the output speed of HMCVT continuously changes from the minimum speed (e.g., 0 ) to the maximum speed. Multistage EGTs or two EGTs with multiple segments (fixed transmission ratio) are often used in HMCVT design to obtain a large transmission ratio range. By reasonably setting the system parameters of EGTs and HST, the multistage shifting transmission ratios are almost the same to reduce the impact. In the shifting process, shift quality is improved by using the control strategy of nonspeed difference. HMCVT technology, with its characteristics of ride comfort and fuel economy, has been widely studied and applied [4-8].

Wang et al. [9], in his research on the mechanical efficiency of HMCVT, performed a theoretical reasoning and calculation of the mechanical efficiency of HMCVT and made a comparative analysis with an improved scheme. Cheng et al. [10] studied, based on the improved simulated annealing algorithm, the efficiency model of the hybrid continuously variable transmission. Li et al. $[11,12]$ analyzed the power distribution of compound and closed planetary gear transmissions and calculated the overall transmission efficiency with and without considering the power loss. The results showed that graphical representation is a practical power analysis and efficiency calculation method that can provide a theoretical reference for the design of complex closed planetary gear transmissions. Awadallah et al. [13] established a simulation model based on the mathematical model of conventional and mild hybrid power systems and studied its transmission efficiency through simulation. 
Although simulation studies have high work efficiency and reliability, they lack experimental verification. In addition, transmission efficiency has consistently been the focus of research on EGT [14-17]. HMCVT has important features and advantages because of the organic combination of EGTs and HST. In the HST system [18, 19], when the working pressure $\Delta_{p}$ is improved, the mechanical efficiency $\eta_{m h}$ of the system is gradually increased, whereas the volumetric efficiency $\eta_{v}$ of the system is reduced. This changing regularity directly affects the working characteristics of HMCVT. Mechanical efficiency $\eta_{m h}$ affects the output torque ratio of the hydraulic system, and volumetric efficiency $\eta_{v}$ affects the output angular velocity ratio. Maintaining HMCVT's continuously variable transmission ratio in the HST system involves two aspects, namely, mechanical efficiency $\eta_{m h}$ and volumetric efficiency $\eta_{v}$, both of which affect the overall efficiency of HMCVT. The effects of mechanical efficiency $\eta_{m h}$ and volumetric efficiency $\eta_{v}$ on the overall efficiency of HMCVT are studied to obtain a relatively uniform efficiency expression. Through numerical examples, this study provides a method to analyze the overall efficiency of HMCVT.

\section{Parameter Matching}

In accordance with the role of EGTs in HMCVT, HMCVTs with three active shafts [1] are divided into input coupled planetary (summing planetary) and output coupled planetary (divider planetary). The single variable hydraulic unit of HST is selected as the foundation of this research to simplify the derivation of the relationship between system parameters. In the system efficiency analysis (Section 5.2), the double-variable hydraulic units of HST are adopted to establish a method of improving system efficiency. As shown in Figure 1, an HMCVT with three active shafts and input coupled planetary is investigated in this work. The power splitting point lies in the intersection of link 1 and shaft I. In accordance with the difference in torques, the shaft throughout transmission system is divided into three links, namely, shaft $I$, link 1 , and link 5 . The power merging point is located between links 4 and 8 . Angular velocities $\omega L(\omega 6)$ and $\omega \mathrm{H}(\omega 7)$ present a low output speed and a high output speed of HMCVT, respectively. In Figure $1, Z i(i=1,2,3,4, s$, $s^{\prime}, p, p^{\prime}$, and $r$ ) is the tooth number for each meshing gear. $Z_{c}$ is the equivalent tooth number of planetary carriers according to the installation diameter of the planetary gears.

Links $4,5,6,7$, and 8 comprise two-stage EGTs (the mechanical path). Link 6 is the planet carrier of EGTs, and link 7 is the sun gear of second-stage EGT. HST (the variable path) is composed of constant displacement $\left(q_{c}\right)$ hydraulic unit 2 and variable displacement $\left(q_{v}\right)$ hydraulic unit 1 , with the former being connected to link 3. Links 1, 2, 3, and 4 comprise the internal transmissions with a fixed ratio.

2.1. Transmission Ratio Calculation. In the transmission scheme shown in Figure 1, the angular velocities of links 1 and 5 are equal to the input angular velocity.

$$
\omega_{1}=\omega_{1}=\omega_{5}
$$

where $\omega_{i}$ denotes the actual angular velocity of the ith link. Displacement ratio $e$ is equal to the displacement of the variable hydraulic unit $\left(q_{v}\right)$ divided by the displacement of the constant unit $\left(q_{c}\right)$, i.e., $e=q_{v} / q_{c}$. The angular velocity ratio of link 3 to link 2 is called the transmission ratio $\tau_{H}$ of the variable path. Given that the constant hydraulic unit acts as a motor or a pump, two expressions can be established as follows [1]:

$$
\tau_{H}=\frac{\omega_{3}}{\omega_{2}}= \begin{cases}\frac{q_{v} \eta_{v}}{q_{c}}=e \eta_{v}, & \text { III (motor), } \\ \frac{q_{v}}{\left(q_{c} \eta_{v}\right)}=\frac{e}{\eta_{v}}, & \text { I (pump). }\end{cases}
$$

The angular velocity ratio of link 2 to link 1 is called the fixed transmission ratio $\tau_{F 1}$, i.e., $\tau_{F 1}=-Z_{1} / Z_{2}$. The angular velocity ratio of link 4 to link 3 is called the fixed transmission ratio $\tau_{F 2}$, that is, $\tau_{F 2}=-Z_{3} / Z_{4}$. By using $\tau_{H}^{\prime}$ to represent the total transmission ratio of parameters $\tau_{F 1}, \tau_{F 2}$ and $\tau_{H}$, we obtain

$$
\tau_{H}^{\prime}=\tau_{H} \tau_{F 1} \tau_{F 2}= \begin{cases}\frac{e \eta_{v} Z_{1} Z_{3}}{\left(Z_{2} Z_{4}\right)}, & \text { III, } \\ \frac{e Z_{1} Z_{3}}{\left(Z_{2} Z_{4} \eta_{v}\right)}, & \text { I. }\end{cases}
$$

The relative angular velocity of the ith link, $\omega_{i}^{j}$, in a reference frame (the $j$ th link) rotating with angular velocity $\omega_{j}$ is defined as

$$
\omega_{i}^{j}=\omega_{i}-\omega_{j}
$$

In the EGTs, $\tau_{P G}$ and $\tau_{P G}^{\prime}$ are defined as the ratio of ring gear angular velocity to sun gear angular velocity when the planetary carrier is fixed for the first and second EGTs, respectively,

$$
\begin{gathered}
\tau_{P G}=\frac{\omega_{4}^{6}}{\omega_{5}^{6}}=-\frac{Z_{s} Z_{p^{\prime}}}{Z_{p} Z_{r}}, \\
\tau_{P G}^{\prime}=\frac{\omega_{4}^{6}}{\omega_{7}^{6}}=-\frac{Z_{s^{\prime}}}{Z_{r}} .
\end{gathered}
$$

In the HMCVT, $\tau_{H M}$ and $\tau_{H M}^{\prime}$ are defined as the ratio of output angular velocity to input angular velocity when $\omega_{L}$ and $\omega_{H}$ are required, respectively,

$$
\begin{aligned}
& \tau_{H M}=\frac{\omega_{L}}{\omega_{I}}=\frac{\tau_{P G}-\tau_{H}^{\prime}}{\tau_{P G}-1}, \\
& \tau_{H M}^{\prime}=\frac{\omega_{H}}{\omega_{I}}=\frac{\left(\tau_{P G}^{\prime}-\tau_{P G}\right) \tau_{H}^{\prime}+\tau_{P G}\left(1-\tau_{P G}^{\prime}\right)}{\tau_{P G}^{\prime}\left(1-\tau_{P G}\right)},
\end{aligned}
$$

where equations (3), (5), and (6) are utilized. We let $k=\left(1-\tau_{P G}\right)-1$ and $k^{\prime}=\left(\tau_{P G^{-}}^{\prime}-\tau_{P G}\right) \quad\left(\tau_{P G}^{\prime}-\tau_{P G}^{\prime} \tau_{P G}\right)-1$; then, equations (7) and (8) can be rewritten as 


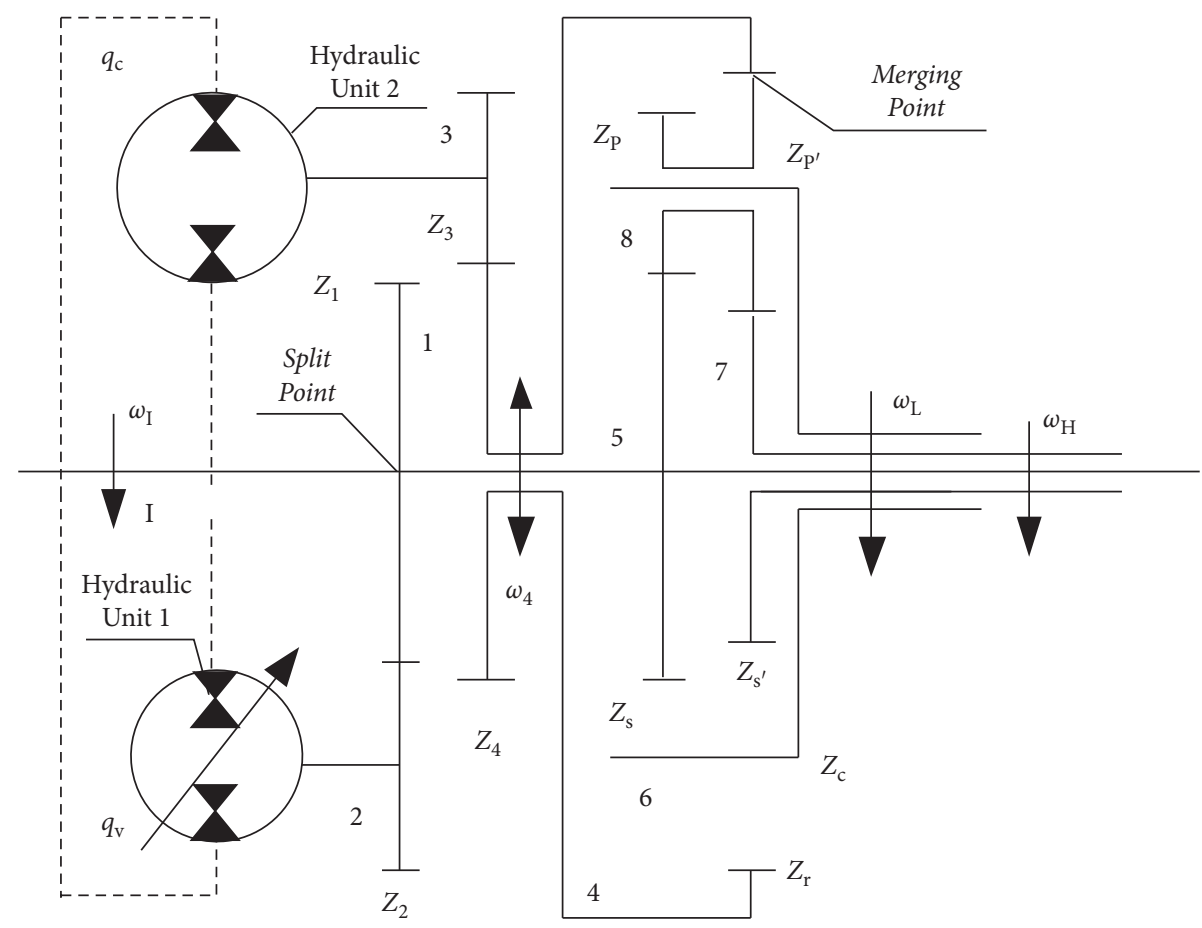

FIGURE 1: Sketch of the transmission system.

$$
\begin{gathered}
\tau_{H M}=k \tau_{H}^{\prime}+1-k, \\
\tau_{H M}=k \tau_{H}^{\prime}+\left(1-k^{\prime}\right) .
\end{gathered}
$$

2.2. Transmission Ratio Requirement. With parameter $k_{H}$ to show the range of variable $\tau_{H}^{\prime}$ and parameter $\lambda$ to represent the asymmetry coefficient of $\tau_{H}^{\prime}\left(k_{H}>0, \lambda>0\right)$, when $\tau_{H}^{\prime}$ increases from $-k_{H}$ to $\lambda k_{H}, \tau_{H M}$ increases gradually within $\left[0, \tau_{H M}^{0}\right]$, where $\tau_{H M}^{0}$ is the maximum of $\tau_{H M}$.

When $\tau_{H}^{\prime}=-k_{H}$ and $\tau_{H M}=0$, we can deduce from equation (7) that $\tau_{P G}=-k_{H}$. When $\tau_{H}^{\prime}=\lambda k_{H}$ and $\tau_{H M}=\tau_{H M}^{0}$, we can deduce from equation (7) that $\tau_{H M}^{0}=(1+\lambda) k_{H} /\left(1+k_{H}\right)$.

When $\tau_{H}^{\prime}$ decreases from $\left[-k_{H}, \lambda_{k H}\right], \tau_{H M}^{\prime}$ increases gradually within $\left[\tau_{H M}^{0}, \tau_{H M}^{\max }\right]$, where $\tau_{H M}^{\max }$ is the maximum of $\tau_{H M}^{\prime}\left(\tau_{H M}^{0}<\tau_{H M}^{\max }\right)$. When $\tau_{H M}^{\prime}=\tau_{H M}^{0}$, we can deduce from equation (8) that

$$
\left\{\begin{array}{l}
\tau_{H M}^{0}=1, \\
k_{H}=\frac{1}{\lambda} .
\end{array}\right.
$$

We can obtain the expression of $\tau_{H M}^{\prime}$ from equation (8) by using equations (10) and (11). $t_{H M}^{\prime}=t_{H M}^{\max }$, and $\tau_{P G}^{\prime}=-1 / \tau_{H M}^{\max }$.

When $\tau_{P G}=-1 / \lambda$ and $\tau_{P G}^{\prime}=-1 / \tau_{H M}^{\max }, \tau_{H}^{\prime}$ gradually increases in the range of $[-1 / \lambda, 1]$. Correspondingly, transmission ratio $\tau_{H M}$ increases from 0 to 1 , and transmission ratio $\tau_{H M}^{\prime}$ decreases from $\tau_{H M}^{\max }$ to 1 . We can deduce from equations (9) and (10) that

$$
\begin{cases}k=\frac{-1}{\tau_{P G}-1}=\frac{\lambda}{\lambda+1}, & 0<k<1, \\ k \prime=\frac{\tau_{P G}^{\prime}-\tau_{P G}}{\tau_{P G}^{\prime}\left(1-\tau_{P G}\right)}=-\frac{\lambda}{\lambda+1}\left(\tau_{H M}^{\max }-1\right) & k^{\prime}<0 .\end{cases}
$$

An example is provided throughout the paper. When $\tau_{F 1} \tau_{F 2}=1, \lambda=1$ and $\tau_{H M}^{\max }=2.7$, with the above mentioned equations, we can obtain

$$
\begin{aligned}
& \left\{\begin{array}{l}
\tau_{P G}=-1, \\
\tau_{P G}^{\prime}=-0.37,
\end{array}\right. \\
& \left\{\begin{array}{l}
\tau_{H M}=0.5 \tau_{H}^{\prime}+0.5, \\
\tau_{H M}^{\prime}=-0.85 \tau_{H}^{\prime}+1.85 .
\end{array}\right.
\end{aligned}
$$

The tooth numbers of the EGTs fitting equations (13) and (14) are summarized in Table 1.

\section{Power Flows in the Absence of Losses}

3.1. Angular Velocity Ratios in the Absence of Losses. In the example mentioned, considering the absence of losses, $\tau_{H}^{\prime}=\tau_{H}=e$. By using equations (9) and (10), the relationship between output angular velocity ratio $\tau_{H M}$ of HMCVT and displacement ratio $e$ is shown in Figure 2(a).

3.2. Direction of Power Flows. According to Macmillan [20], the direction of power flow in EGT cannot be modified by meshing friction. Torque equilibrium and power conservation equations are widely used to determine the efficiency 
TABLe 1: Tooth numbers of the planetary gear train.

\begin{tabular}{llllll}
\hline$Z_{s}$ & $Z_{r}$ & $Z_{c}$ & $Z_{p}$ & $Z_{s^{\prime}}$ & $Z_{p^{\prime}}$ \\
\hline 48 & 92 & 63 & 15 & 34 & 29 \\
\hline
\end{tabular}

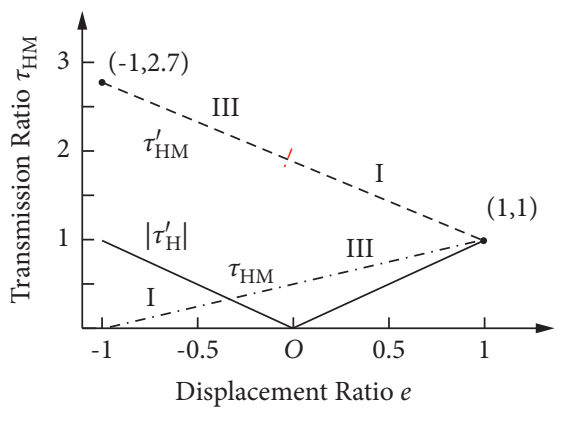

(a)

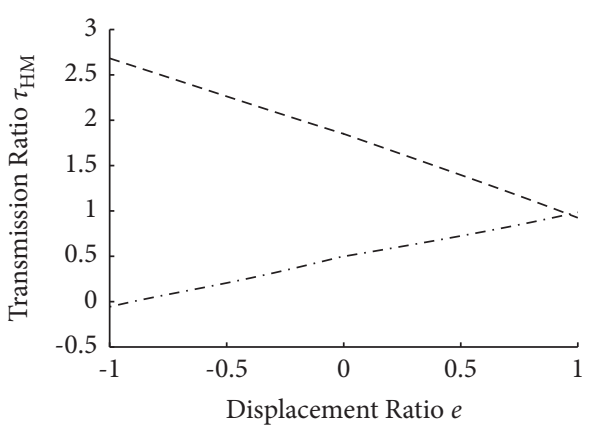

(b)

FIgURE 2: Relationship between output transmission ratio $\tau_{H M}$ and displacement ratio $e$. (a) In the absence of losses. The working area of $\tau_{H M}$ is formed by dot-dashed and double-dot-dashed lines, and the working area of $\tau_{H M}^{\prime}$ is formed by dashed and long dashed lines. The solid line is $\left|\tau_{H}^{\prime}\right|$. HMCVT realizes continuously variable transmission in the range of $[0,2.7]$, shifting with no speed difference in point $(1,1)$. (b) With the losses calculated by Sts1 in Section 5.2.

of EGT $[1,21]$. In this study, the power that flows into EGT is positive, and the outflow is negative.

On steady state, directions of the possible power flow [22-24] are those shown in Figure 3 as type I, type II, and type III flow.

When power conservation and torque equilibrium equations are applied to EGT, we can obtain the following from Figure 3:

$$
\begin{array}{r}
T_{O} \omega_{O}+T_{4} \omega_{4}+T_{5} \omega_{5}=0 \\
T_{O}+T_{4}+T_{5}=0
\end{array}
$$

where $T_{i}$ is the torque applied on the ith link. Without loss of generality, we can assume that the output shaft of the EGTs is link 7. In this case, by using equations (1) and (10), we obtain

$$
\begin{aligned}
& \omega_{O}=\omega_{I} \tau_{H M}^{\prime}=\omega_{4} k^{\prime}+\omega_{5}\left(1-k^{\prime}\right), \\
& \frac{T_{7}}{-1}=\frac{T_{4}}{k^{\prime}}=\frac{T_{5}}{1-k^{\prime}} .
\end{aligned}
$$

In type I power flow with output shaft 7 [22], equation (15) is rewritten as

$$
\left|T_{7} \omega_{7}\right|+\left|T_{4} \omega_{4}\right|-\left|T_{5} \omega_{5}\right|=0 .
$$

By substituting equations (10), (18), and (17) in equation (19), we obtain

$$
\left|\tau_{H}^{\prime}\right|=\left|\frac{1-k^{\prime}}{k^{\prime}}\right|-\frac{1}{\left|k^{\prime}\right|}\left|\tau_{H M}^{\prime}\right|
$$

As indicated in the first quadrant of Figure 2(a), equation (10) is rewritten as

$$
\left|\tau_{H M}^{\prime}\right|=\left|1-k^{\prime}\right|-\left|k^{\prime} \tau_{H}^{\prime}\right| \quad k^{\prime}<0 \tau_{H}^{\prime}>0 .
$$

In consideration of equations (18) and (21), equation (20) is identical. When the output shaft is link 7, the work area for generating type I power flow is shown in the first quadrant of Figure 2(a) by long dashed lines.

The direction of power flow relative to every working condition can be obtained. Figure 2(a) shows every type of power flow with symbols I and III.

PM is the HST system (the variable path). PG is the twostage EGTs (the mechanical path). F1 and F2 are the internal transmissions with a fixed ratio. $1,2,3,4,5, \mathrm{I}$, and $\mathrm{O}$ are the links of HMCVT shown in Figure 1.

\section{Efficiency of HMCVT}

4.1. Torque Ratio of Links with Losses. The pressure efficiency $\eta \mathrm{p}$ of the HST system is approximately expressed as $\eta \mathrm{p}=p_{P} /$ $p_{M}$, where $p_{P}$ and $p_{M}$ are pump and motor operating pressures, respectively. In the HST system, total mechanical efficiency $\eta_{m h}$ is expressed as $\eta_{m h}=\eta_{p} \eta_{P} \eta_{M}$, where $\eta_{M}$ and $\eta_{P}$ are pump and motor mechanical efficiencies, respectively. The output torque of motor $T_{M}$ and the input torque of pump $T_{P}$ are expressed as

$$
\begin{aligned}
T_{P} & =\frac{q_{P} p_{P}}{2 \pi \eta_{P}}, \\
T_{M} & =\frac{q_{M} p_{M} \eta_{M}}{2 \pi},
\end{aligned}
$$

where $q_{P}$ and $q_{M}$ are the displacements of the pump and motor, respectively.

In type III power flow, hydraulic unit 1 acts as the variable pump with a torque of $T_{P}$, and hydraulic unit 2 acts 


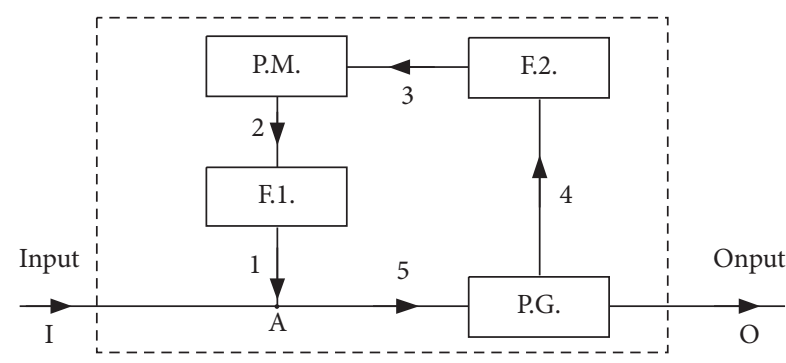

(a)

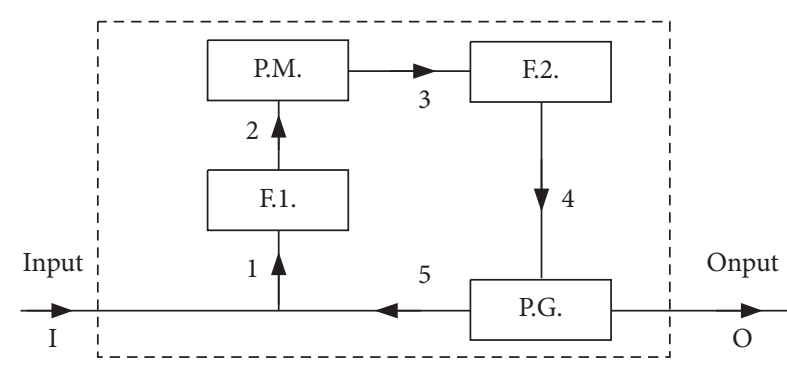

(b)

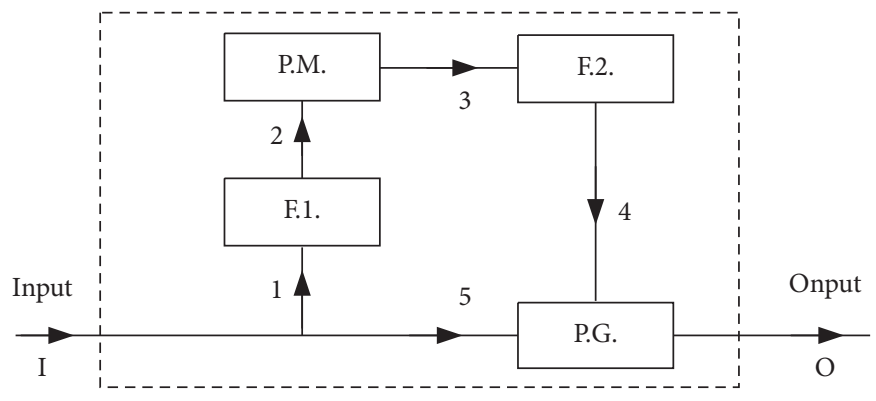

(c)

Figure 3: Power flows in HMCVT. (a) Type I power flow. (b) Type II power flow. (c) Type III power flow.

as the motor with a torque of $T_{M}$. The torque ratio $\Delta_{H}$ of HST in type III power flow is defined as $T_{P}$ divided by $T_{M}$.

$$
\Delta_{H}=\frac{T_{P}}{T_{M}}=\left|\frac{T_{2}}{T_{3}}\right|=\left|\frac{q_{P} p_{P}}{q_{M} p_{M} \eta_{M} \eta_{P}}\right| \approx \frac{|e|}{\eta_{M} \eta_{P} \eta_{p}}=\frac{|e|}{\eta_{m h}} .
$$

The torque ratio $\Delta_{12}$ of F1 to F2 (shown in Figure 3; internal transmissions) in type III power flow is defined as

$$
\Delta_{12}=\frac{\tau_{F 1} \tau_{F 2}}{\eta_{F 1} \eta_{F 2}}
$$

In type I power flow, hydraulic unit 1 acts as the variable motor with a torque of $T_{M}$, and hydraulic unit 2 acts as the pump with a torque of $T_{P}$. $\Delta_{H}^{\prime}$ is defined as $T_{P}$ divided by $T_{M}$.

$$
\Delta_{H}^{\prime}=\frac{T_{P}}{T_{M}}=\left|\frac{T_{3}}{T_{2}}\right|=\left|\frac{q_{P} p_{P}}{q_{M} p_{M} \eta_{M} \eta_{P}}\right| \approx \frac{1}{|e| \eta_{M} \eta_{P} \eta_{p}}=\frac{1}{|e| \eta_{m h}} .
$$

The torque ratio $\Delta_{12}^{\prime}$ of F1 to F2 in type I power flow is defined as

$$
\Delta_{12}^{\prime}=\frac{1}{\eta_{F 1} \eta_{F 2} \tau_{F 1} \tau_{F 2}}
$$

Given that the transmission losses and the torques applied to the links are unrelated to the observer's motion, the method of epicyclic inversion was used by Pennestri and Freudenstein [14] and Chen and Liang [21] in different ways.

This study uses the transforming mechanism model $[21,25]$, in which the interaction forces and the relative angular velocity are all the same as the EGTs shown in
Figure 1, but the planet carrier is fixed. Thus, the losses in the two models are considered to be the same. The torque ratio of the links based on meshing power [21, 25] is applied to calculate the efficiency of the two-DOF EGTs. Meshing power $p_{i}^{c}$ (the ith link relative to planet carrier 6) is a virtual power that is independent of the actual power $p_{i}$. Meshing power ratio $\psi_{i}$ is defined as the ratio of meshing power $p_{i}^{c}$ through the ith link to actual power $p_{i}$ through the same link. If power pi flows into EGT, it is defined as positive (negative otherwise). The expressions of $\omega_{i}^{6}, p_{i}^{6}, p_{i}$ and $\psi_{i}$ are

$$
\left\{\begin{array}{l}
\omega_{i}^{6}=\omega_{i}-\omega_{6}, \\
p_{i}^{6}=T_{i}\left(\omega_{i}-\omega_{6}\right)=T_{i} \omega_{i}^{6}, \\
p_{i}=T_{i} \omega_{i}, \\
\psi_{i}=\frac{p_{i}^{6}}{i}=\frac{T_{i}\left(\omega_{i}-\omega_{6}\right)}{T_{i} \omega_{i}}=\frac{\omega_{i}^{6}}{\omega_{i}} .
\end{array}\right.
$$

In the transforming mechanism, the direction of $p_{i}^{\mathrm{c}}$ can be determined in accordance with the sign of $p_{i}$ and $\psi_{i}$. If the sign of $p_{i}^{c}$ is positive, power $p_{i}^{c}$ flows into the transforming mechanism; otherwise, it flows out of the system.

Without loss of generality, we assume that the output shaft of the HMCVT is link 7 in type I power flow, and the relationship among $\omega_{i}, \psi_{i}$ and $p_{i}$ between links is $\omega_{7}>\omega_{5}>\omega_{6}>\omega_{4}>0$. 


$$
\begin{cases}\psi_{5}=\frac{p_{5}^{6}}{p_{5}}=\frac{T_{5}\left(\omega_{5}-\omega_{6}\right)}{T_{5} \omega_{5}}>0, & p_{5}<0, p_{5}^{6}>0, \\ \psi_{4}=\frac{p_{4}^{6}}{p_{4}}=\frac{T_{4}\left(\omega_{4}-\omega_{6}\right)}{T_{4} \omega_{4}}<0, & p_{4}<0, p_{4}^{6}>0, \\ \psi_{7}=\frac{p_{7}^{6}}{p_{7}}=\frac{T_{7}\left(\omega_{7}-\omega_{6}\right)}{T_{7} \omega_{7}}>0, & p_{7}<0, p_{7}^{6}<0 .\end{cases}
$$

Under this condition, the meshing power is from links 5 and 4 to link 7 in the transforming mechanism. When the power conservation equation and the torque equilibrium condition are applied to the EGT, we have

$$
\left\{\begin{array}{l}
T_{4} \omega_{4}^{6} \eta_{48}^{6} \eta_{87}^{6}+T_{5} \omega_{5}^{6} \eta_{58}^{6} \eta_{87}^{6}+T_{7} \omega_{7}^{6}=0, \\
T_{4}+T_{5}+T_{7}=0
\end{array}\right.
$$

where $\eta_{i j}^{6}$ is the efficiency of the path, in which the meshing power flows from the $i$ th link to the jth link with the planet carrier being fixed in the transforming mechanism.

To obtain the relationship between output torque and input torque, by using equations (5) and (6), we can reform equation (29) to

$$
\left\{\begin{array}{l}
T_{7}=T_{5} \frac{\eta_{48}^{6} \eta_{87}^{6} \tau_{P G}^{\prime} \tau_{P G}-\eta_{58}^{6} \eta_{87}^{6} \tau_{P G}^{\prime}}{\left(1-\eta_{48}^{6} \eta_{87}^{6} \tau_{P G}^{\prime}\right) \tau_{P G}} \\
T_{4}=-T_{5}\left(1+\frac{\eta_{58}^{6} \eta_{87}^{6} \tau_{P G}^{\prime} \tau_{P G}-\eta_{58}^{6} \eta_{87}^{6} \tau_{P G}^{\prime}}{\left(1-\eta_{48}^{6} \eta_{87}^{6} \tau_{P G}^{\prime}\right) \tau_{P G}}\right)
\end{array}\right.
$$

From equation (28), torque ratios $\Delta_{75}$ and $\Delta_{45}^{\prime}$ of EGT (shown in Figure 3; the mechanical path) in type I power flow are defined as

$$
\left\{\begin{array}{l}
\Delta_{75}=\frac{\eta_{48}^{6} \eta_{87}^{6} \tau_{P G}^{\prime} \tau_{P G}-\eta_{58}^{6} \eta_{87}^{6} \tau_{P G}^{\prime}}{\left(1-\eta_{48}^{6} \eta_{87}^{6} \tau_{P G}^{\prime}\right) \tau_{P G}} \\
\Delta_{45}^{\prime}=-\left(1+\frac{\eta_{48}^{6} \eta_{87}^{6} \tau_{P G}^{\prime} \tau_{P G}-\eta_{58}^{6} \eta_{87}^{6} \tau_{P G}^{\prime}}{\left(1-\eta_{48}^{6} \eta_{87}^{6} \tau_{P G}^{\prime}\right) \tau_{P G}}\right)
\end{array}\right.
$$

When the output shaft of the EGT is link 7 in type III power flow, in reference to the actual angular velocity $\omega_{i}$, the relationship between links is $\omega_{7}>\omega_{5}>\omega_{6}>0>\omega_{4}$. The same expression of $\Delta_{75}$ and $\Delta_{45}^{\prime}$ as that in equation (29) can be obtained because the sign of $p_{i}^{c}$ remains similar to that in type I power flow. The other case is that the output shaft of HMCVT is link 6 in types III and I power flows. In this condition, we can obtain the expressions of $\Delta_{65}$ and $\Delta_{45}$ in a similar manner.

$$
\left\{\begin{array}{l}
\Delta_{45}=-\frac{\eta_{58}^{6} \eta_{84}^{6}}{\tau_{P G}} \\
\Delta_{65}=\frac{\eta_{58}^{6} \eta_{84}^{6}}{\tau_{P G}}-1
\end{array}\right.
$$

4.2. Efficiency of HMCVT. In the steady state of HMCVT, we assume that output torque To of HMCVT is the result of the calculation and not of the actual load, such that the input torque and angular velocity remain similar to those for diesel working under rated conditions. When the power pi contributed to $T_{i}$ flows into the Split point in Figure 1, $T_{i}$ is defined as positive; otherwise, it is negative. Here, $T_{5}$ is negative in type I and III power flows. To maintain consistency with the signs of $T_{5}$ and $T_{1}$ for EGTs, when the torque equilibrium condition is applied to the Split point, we have

$$
T_{I}-T_{5}-T_{1}=0 .
$$

Depending on the type of power flow with output shaft 6 , two kinds of relationship exist between $T_{1}$ and $T_{5}$.

$$
T_{1}= \begin{cases}\Delta_{H}\left|\Delta_{45}\right| \Delta_{12} T, & \text { III, } \\ -\frac{\left|\Delta_{45}\right|}{\Delta_{H}^{\prime} \Delta_{12}^{\prime}} T, & \text { I. }\end{cases}
$$

The relationship between $T_{5}$ and $T_{\mathrm{I}}$ is

$$
T_{5}= \begin{cases}\frac{T_{I}}{1+\Delta_{H}\left|\Delta_{45}\right| \Delta_{12}}, & \text { III, } \\ \frac{T_{I}}{1-\left|\Delta_{45}\right| / \Delta_{H}^{\prime} \Delta_{12}^{\prime}}, & \text { I. }\end{cases}
$$

We obtain the transmission efficiency of the HMCVT with output shaft 6 by using the relationship expressions of $T_{I}$ with $T_{5}$ and equation (7) as follows:

$$
\eta_{H M}=-\frac{T_{6} \omega_{6}}{T_{I} \omega_{I}}=\left\{\begin{array}{l}
-\frac{\Delta_{65} T_{I} \omega_{6} /\left(1+\Delta_{H}\left|\Delta_{45}\right| \Delta_{12}\right)}{T_{I} \omega_{I}}=\frac{-\Delta_{65} \tau_{H M}}{1+\Delta_{H}\left|\Delta_{45}\right| \Delta_{12}}, \\
-\frac{\Delta_{65} T_{I} \omega_{6} /\left(1-\left(\left|\Delta_{45}\right| / \Delta_{H}^{\prime} \Delta_{12}^{\prime}\right)\right)}{T_{I} \omega_{I}}=\frac{-\Delta_{65} \tau_{H M}}{1-\left(\left|\Delta_{45}\right| / \Delta_{H}^{\prime} \Delta_{H}^{\prime}\right)}, \quad \text { I. }
\end{array}\right.
$$


The working pressure of the hydraulic system is represented by the working pressure of the fixed displacement hydraulic unit 2.

$$
\Delta p=\frac{2 \pi\left|T_{3}\right|}{q_{c}}= \begin{cases}\frac{2 \pi\left|\tau_{F 2} \Delta_{45}\right| T_{5}}{q_{c} \eta_{F 2}}=\frac{2 \pi\left|\tau_{F 2} \Delta_{45}\right| T_{I}}{q_{c} \eta_{F 2}\left(1+\left|\Delta_{45}\right| \Delta_{12} \Delta_{H}\right)}, & \text { III, } \\ \frac{2 \pi\left|\tau_{F 2} \Delta_{45}\right| T_{5} \eta_{F 2}}{q_{c}}=\frac{2 \pi\left|\tau_{F 2} \Delta_{45}\right| \eta_{F 2} T_{I}}{q_{c}\left(1-\left(\left|\Delta_{45}\right| / \Delta_{12}^{\prime} \Delta_{H}^{\prime}\right)\right)}, \quad \text { I. } & \text {. }\end{cases}
$$

When the output shaft is link 7, equations (32), (34), and (35) remain the same, but $\Delta_{45}, \Delta_{65}$ and $\tau_{H M}$ are replaced by $\Delta_{45}^{\prime}, \Delta_{75}$, and $\tau_{H M}^{\prime}$, respectively:

$$
\begin{aligned}
& T_{1}= \begin{cases}\Delta_{\mathrm{H}}\left|\Delta_{45}^{\prime}\right| \Delta_{12} T_{5}, & \text { III, } \\
-\frac{\left|\Delta_{45}^{\prime}\right|}{\Delta_{H}^{\prime} \Delta_{12}^{\prime}} T_{5}, & \text { I, }\end{cases} \\
& \eta_{H M}^{\prime}=-\frac{T_{7} \omega_{7}}{T_{\mathrm{I}} \omega_{\mathrm{I}}}= \begin{cases}\frac{-\Delta_{75} \tau_{H M}^{\prime}}{1+\Delta_{\mathrm{H}}\left|\Delta_{45}^{\prime}\right| \Delta_{12}}, & \text { III, } \\
\frac{-\Delta_{75} \tau_{H M}^{\prime}}{1-\left(\left|\Delta_{45}^{\prime}\right| / \Delta_{H}^{\prime} \Delta_{12}^{\prime}\right)}, & \text { I, }\end{cases} \\
& \Delta^{\prime} p=\frac{2 \pi\left|T_{3}\right|}{q_{c}}= \begin{cases}\frac{2 \pi\left|\Delta_{45}^{\prime} \tau_{F 2}\right| T_{I}}{q_{c} \eta_{F 2}\left(1+\left|\Delta_{45}^{\prime}\right| \Delta_{12} \Delta_{H}\right)}, & \text { III, } \\
\frac{2 \pi\left|\Delta_{45}^{\prime} \tau_{F 2}\right| \eta_{F 2} T_{I}}{q_{c}\left(1-\left(\left|\Delta_{45}^{\prime}\right| / \Delta_{12}^{\prime} \Delta_{H}^{\prime}\right)\right)} & \text { I. }\end{cases}
\end{aligned}
$$

$$
\begin{gathered}
\eta_{H}=\left(\Delta p^{2} \Delta p 1\right) \cdot\left(\begin{array}{ccc}
-0.000203 & 0.000244 & -0.000402 \\
0.010453 & -0.011201 & 0.022677 \\
-0.376406 & 0.618912 & 0.267755
\end{array}\right)\left(e^{2} e 1\right)^{T}, \\
\eta_{v}=\left(\Delta p^{2} \Delta p 1\right) \cdot\left(\begin{array}{ccc}
-0.000145 & 0.000263 & -0.000143 \\
-0.000594 & 0.001655 & -0.002349 \\
0.001936 & -0.008338 & 0.998824
\end{array}\right)\left(e^{2} e 1\right)^{T}, \\
\eta_{m h}=\left(\Delta p^{2} \Delta p 1\right) \cdot\left(\begin{array}{ccc}
-0.000039 & -0.000055 & -0.000251 \\
0.008926 & -0.008787 & 0.023435 \\
-0.377170 & 0.621347 & 0.263924
\end{array}\right)\left(e^{2} e 1\right)^{T} .
\end{gathered}
$$

The analysis steps (Sts1) for obtaining the HMCVT efficiency with the single variable unit are shown below, and the results are listed in Figure 4 and Table 3.

(1) Two curved surfaces in the same coordinate system are established. One is the curved surface of the hydraulic system's mechanical efficiency, and the other is the mechanical system demanding surface.
When the losses from the churning of the lubricating oil and shaft bearing friction are not considered, once the characteristics of the HMCVT have been determined, torque ratios $\Delta_{12}, \Delta_{12}^{\prime}, \Delta_{75}, \Delta_{45}^{\prime}, \Delta_{65}$, and $\Delta_{45}$ become constant. However, firstly, torque ratios $\Delta_{H}$ and $\Delta_{H}^{\prime}$ vary with the change in mechanical efficiency $\eta_{m h}$ and displacement ratio $e$ for the hydraulic system. Secondly, $\tau_{H M}$ and $\tau_{H M}^{\prime}$ vary with the change in volumetric efficiency $\eta_{v}$ and displacement ratio $e$. Thirdly, volumetric efficiency $\eta_{v}$ and mechanical efficiency $\eta_{m h}$ change with displacement ratio $e$ and system working pressure $\Delta_{p}$. According to equation (37) or (40), the curved surface of $\eta_{m h}$ relative to $e$ and $\Delta_{p}$ is called the mechanical system demanding surface.

\section{Efficiency Analysis}

5.1. Numerical Example. The mechanical parameters of the example are displayed in equations (13) and (14) and Table 1. The loss of a conventional gear pair is estimated via the following formula [13]:

$$
\psi_{m n}^{\mathrm{C}}=0.23\left(\frac{1}{Z_{1}} \pm \frac{1}{Z_{2}}\right),
$$

where $\psi_{m n}^{\mathrm{C}}$ is the meshing loss of the power flowing through the $m$ th and $n$th links when the gear carrier is fixed and $Z_{1}$ and $Z_{2}$ are the teeth numbers of the small and large gears of this pair, respectively.

By using the equations above, we can obtain constant torque ratios. The parameters of the example are listed in Table 2.

By using the hydraulic parameters $\eta_{H}, \eta_{v}$ and $\eta_{m h}$ relative to $\Delta_{p}$ and $e$ [26] listed in Tables 1 and 2, the expressions of the fitting surfaces are obtained, with the displacement of hydraulic unit 2 being fixed to the maximum value. 
TABle 2: Parameters of HMCVT.

\begin{tabular}{lcccc}
\hline$\Delta_{12}$ & $\Delta_{12}^{\prime}$ & $\Delta_{75}$ & $\Delta_{45}^{\prime}$ & $\Delta_{65}$ \\
\hline 1.02 & 1.02 & -0.528 & -0.472 & -1.975 \\
$\Delta_{45}$ & $\tau_{F 2}$ & $T_{I}(\mathrm{~N} \cdot \mathrm{m})$ & $q_{c}\left(\mathrm{~cm}^{3} \cdot \mathrm{r}^{-1}\right)$ & 107 \\
0.975 & 0.729 & 702.5 & $\Delta_{45}$ & 0.975 \\
\hline
\end{tabular}

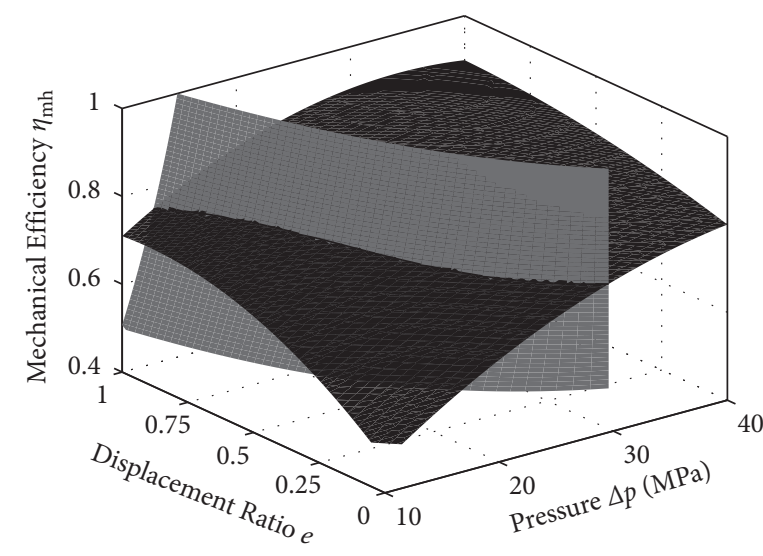

(a)

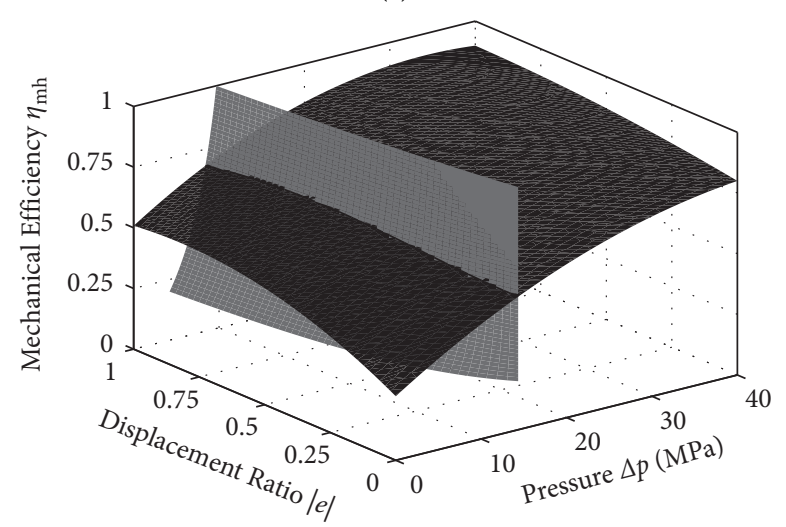

(c)

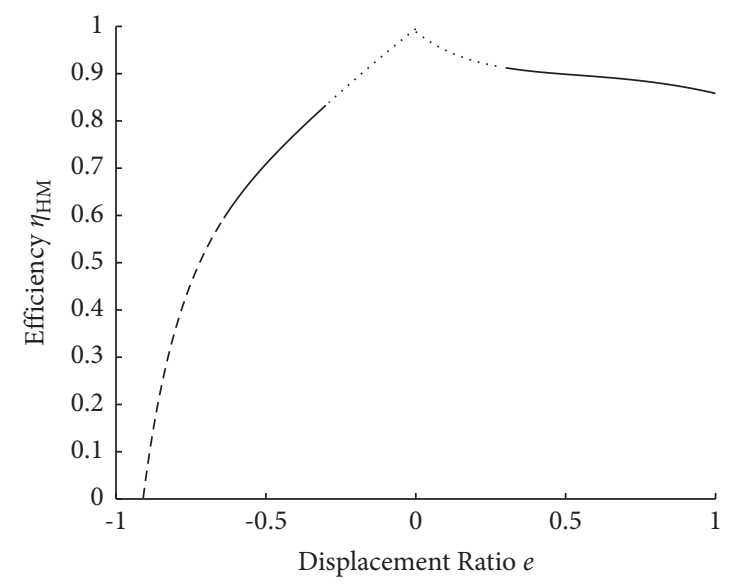

(e)

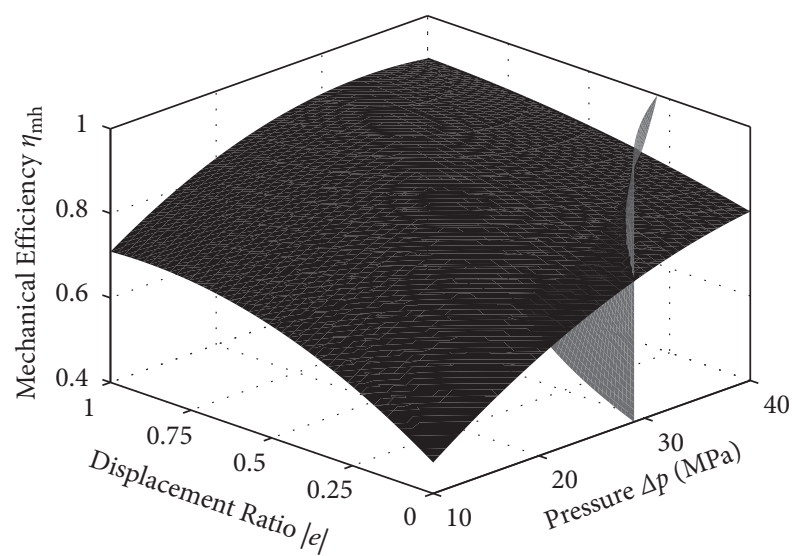

(b)

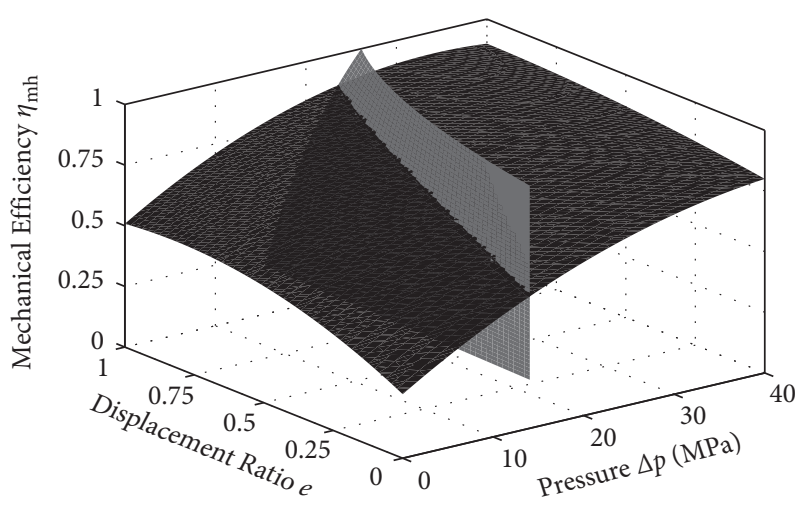

(d)

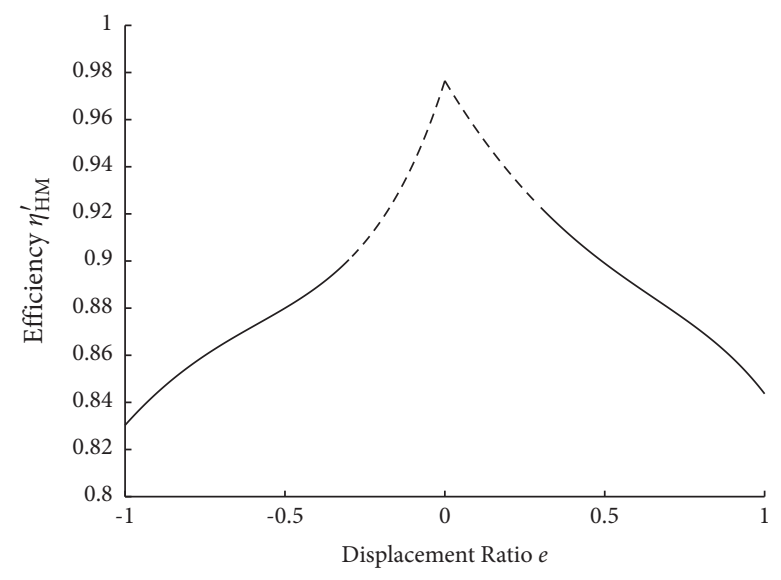

(f)

FIGURE 4: Results of the efficiency analysis. (a) Intersection of surfaces in type III power flow with output shaft 6. (b) Intersection of surfaces in type I power flow with output shaft 6. (c) Intersection of surfaces in type III power flow with output shaft 7. (d) Intersection of surfaces in type I power flow with output shaft 7. (e) Overall efficiency of HMCVT. (f) Overall efficiency of HMCVTwith output shaft 7. 
TABle 3: Polynomial coefficients of ratios.

\begin{tabular}{lcc}
\hline Type & $\Delta_{H}$ or $1 / \Delta_{H}^{\prime}$ & $\tau_{H}$ \\
\hline 6 III $\Delta_{H}$ & {$[0.3515,-0.4770,1.4554,-0.0042]$} & {$[-0.1093,0.2033,0.8792,-0.0027]$} \\
6 I $1 / \Delta_{H}^{\prime}$ & {$[-0.0908,0.1886,0.8034,-0.0028]$} & {$[-0.4537,0.6241,-0.0107,-1.2757,0.0027]$} \\
7 III $\Delta_{H}^{\prime}$ & {$[0.0112,0.6150,-0.9826,1.8224,0.0002]$} & {$[0.0324,-0.0628,-0.9496,0.0005]$} \\
7 I $1 / \Delta_{H}^{\prime}$ & {$[-0.1881,0.5067,0.5532,-0.0005]$} & {$[0.0497,-0.0399,-0.0171,1.0616,0.0002]$} \\
\hline
\end{tabular}

$\Delta_{p}$ relative to $|\mathrm{e}|$ in the fitting formula for $\eta_{v}$, the relationship between $\eta_{v}$ and $\tau_{H}$ with $|e|$ is obtained.

(3) The overall angular velocity ratios $\tau_{H M}$ and $\tau_{H M}^{\prime}$ of the HMCVT are obtained.

(4) The overall efficiencies $\eta_{H M}$ and $\eta_{H M}^{\prime}$ of the HMCVT are obtained.

By observing the overall efficiency of output shaft 6 in Figure 4(e), we find that the least efficiency appears at the start of the vehicle, and $-1<e<-0.9$. The actual numerical result is negative. As shown in Figure 5(b), when $-1<e<-0.9, \tau_{H}<-1$. In this condition, the direction of output shaft 6 is reversed. Therefore, we can assume that the vehicle should be started at an $e$ of about -0.9 , and |e| should be gradually reduced to improve the speed of the vehicle.

(1) The dotted line indicates that the curve is out of the fitting data source. (2) Denoted by a line of long dashes, this working area without the full input power is called traction limiting, indicating that the efficiency curve is highly inaccurate at the start of the entire machine. It is calculated with a peak pressure of $40 \mathrm{MPa}$ of the hydraulic system.

When the vehicle speed is lower than $6 \mathrm{~km} / \mathrm{h}$, the working condition without the full input power is called traction limiting. When $\Delta_{p}>40 \mathrm{MPa}$ in type I power flow with output shaft 6 , the efficiency is calculated using the peak pressure (40 MPa) of the hydraulic system, as shown in Figures 4(b) and 5(a). This kind of calculation reflects the carrying capacity of HMCVT, which is independent of the weight of the whole vehicle and the ground adhesion performance.

In the steady state, with the vehicle speed being larger than the maximum speed in type I power flow with output shaft 6 (about $7.4 \mathrm{~km} / \mathrm{h}$ in the example), the maximum working pressure of the hydraulic system is $29.57 \mathrm{MPa}$ when the system output shaft is link 6 and $e=0$. Considering that the coefficient of torque reservation for the diesel engine is about $40 \%$, when the system is working under rated conditions, the suitable hydraulic system pressure is about $30 \mathrm{MPa}$.

By substituting the polynomials for $\tau_{H}$ (in Table 3 ) in equation (14), the relationship between angular velocity ratio $\tau_{H M}$ of HMCVT and displacement ratio $e$ is obtained, as shown in Figure 2(b). When the system output shaft is link 7, the hydraulic system pressure is generally lower than that for output link 6 because torque ratio $\Delta_{45}^{\prime}$ is smaller than $\Delta_{45}$. The output angular velocity of link 7 is greater than that of link 6, as shown in Figure 2(b).

5.2. HMCVT with Double-Variable Hydraulic Units. In the HMCVT system with double-variable hydraulic units, hydraulic unit 2 is the variable displacement $\left(q_{v}^{\prime} \mathrm{q}^{\prime} \mathrm{v}\right)$ hydraulic unit connected to link 3 in Figure 1. We can obtain a relatively stable $e$ through different combinations of variable qv and variable $q_{v}^{\prime}$, which allow hydraulic units 1 and 2 to work in a relatively high-efficiency area. Without consideration of fuel consumption and vehicle dynamic characteristics, the problem of HMCVT efficiency changes. How variables qv and $q_{v}^{\prime}$ should be varied to obtain the maximum system efficiency in the steady state under the rated conditions of the diesel engine is the new problem to be solved.

The analysis steps (Sts2) for obtaining the curve of variables $q_{v}^{\prime}\left(q_{v}=q_{v}^{\prime} e\right)$ are shown as follows, and the results obtained with the example above are listed in Figure 6:

(1) When variable $e=$ constant, $e^{\prime}=q_{v}^{\prime} / q_{v \max }^{\prime}$, where $q_{v \max }^{\prime}$ is the maximum value of variable $q_{v}^{\prime}$. The fitting formulas (curved surfaces) of $\eta_{\nu}$ and $\eta_{m h}$ relative to $\Delta_{p}$ and $e^{\prime}$ are obtained with the changed displacement of hydraulic unit 2 .

(2) To obtain the relationship between overall efficiency $\eta$ and variable $e^{\prime}$, the steps of Sts 1 are completed with $q_{v}^{\prime}$ replacing $q_{c}$. Attention is given to the difference between $e$ and $e^{\prime}$.

(3) In the range of variable $e$, processes (1) and (2) are repeated. In the three-dimensional coordinate system, the curved surface that represents $\eta$ relative to $e$ and $e^{\prime}$ is obtained, and the points $\left(e, e^{\prime}, \eta\right)$ that have the highest efficiency for each $e$ in step (b) are connected.

By substituting the polynomial $\Delta_{H}^{\prime}$ or $\Delta_{H}$, equations (30) and (31) in equation (34), we obtain the relationship between output torque $T_{o}$ of HMCVT and the foreword speed of the vehicle, whilst assuming that the minimum speed is 0 , and the maximum speed is $39.96 \mathrm{~km} / \mathrm{h}$, as shown in Figure 6(h).

When the machine is started with type I power flow with output shaft 6 , most of the time, it works on $e^{\prime}=1$ or $\Delta^{\prime} p=40 \mathrm{MPa}$, and the efficiency curve is almost the same as that of the single variable unit system. In addition, from the comparisons of the efficiency curves with outputs 6 and 7 in Figures 6(e) and 6(f), we find that the overall efficiency of HMCVT with double-variable hydraulic units is improved. The maximum value of $q_{v}^{\prime}$ is about $91 \mathrm{~cm}^{3} \cdot \mathrm{r}^{-1}$, and the system variable $q_{v}^{\prime}$ is effectively reduced.

5.2.1. The Solid Curves Belong to the HST with a Single Variable. As shown by the curves of $\Delta_{p}$ relative to $e$, the HST of HMCVT always works at the medium-pressure stage, in which the relative maximum efficiency of the hydraulic system can be obtained. However, the working pressure is unstable, mainly because the maximum values 


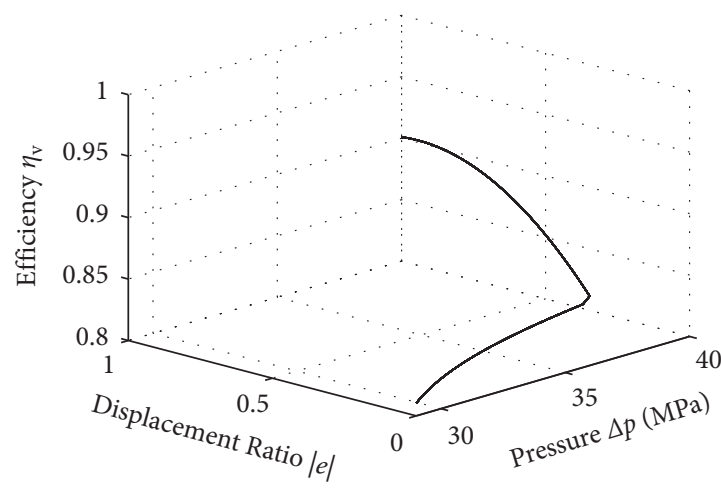

(a)

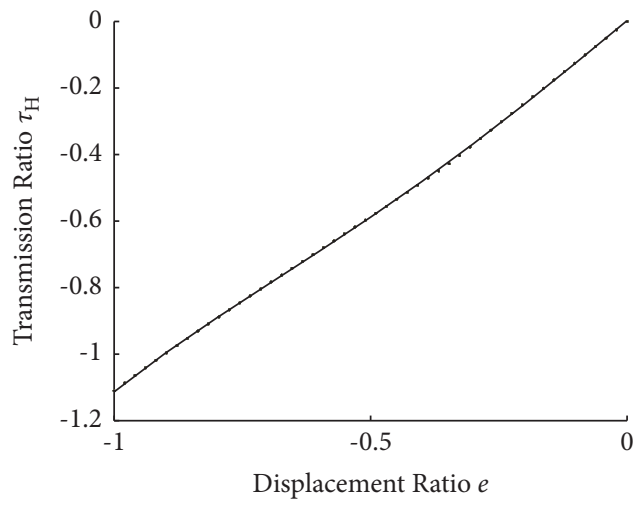

(b)

FIgURE 5: Curves $\eta_{v}$ and $\tau_{H}$ of the numerical results for type I power flow with output shaft 6 .

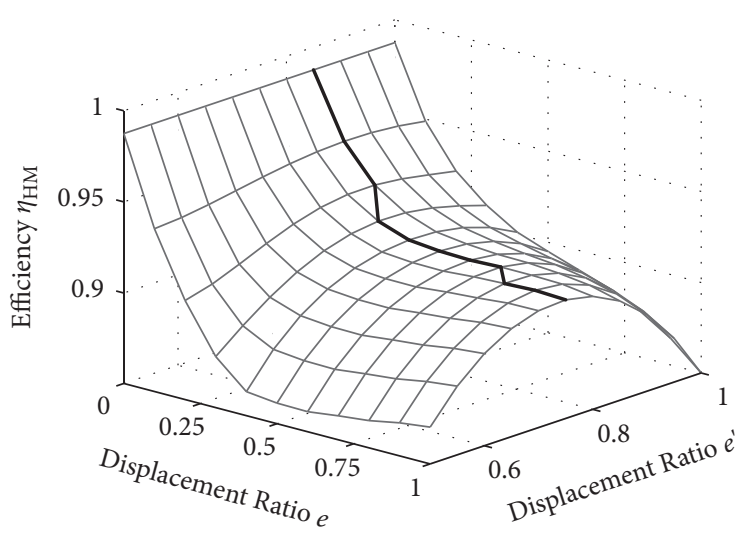

(a)

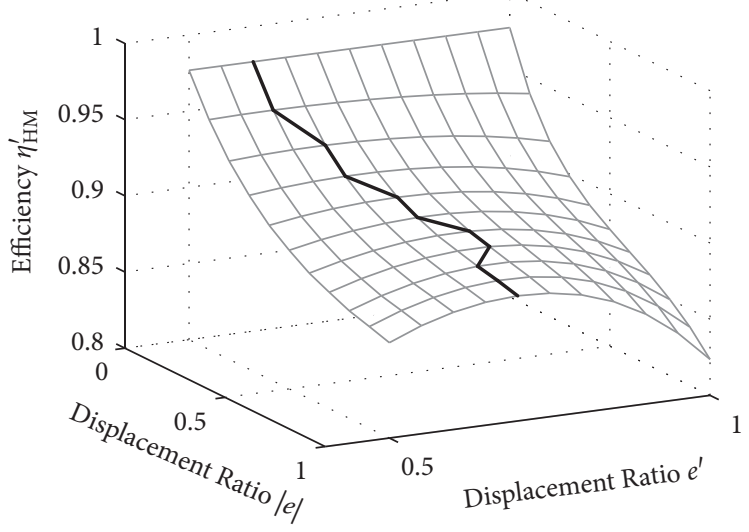

(c)

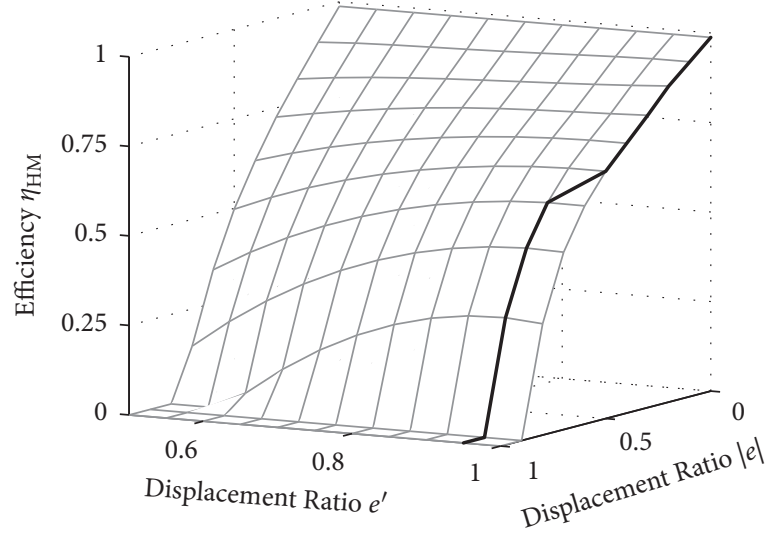

(b)

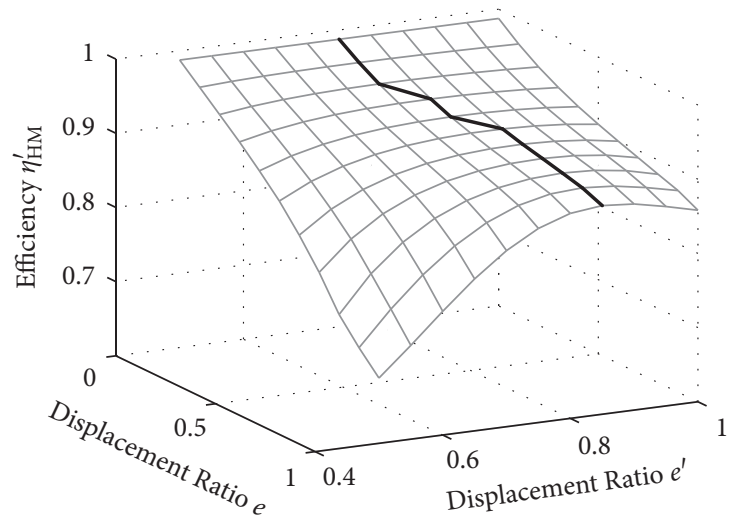

(d)

Figure 6: Continued. 


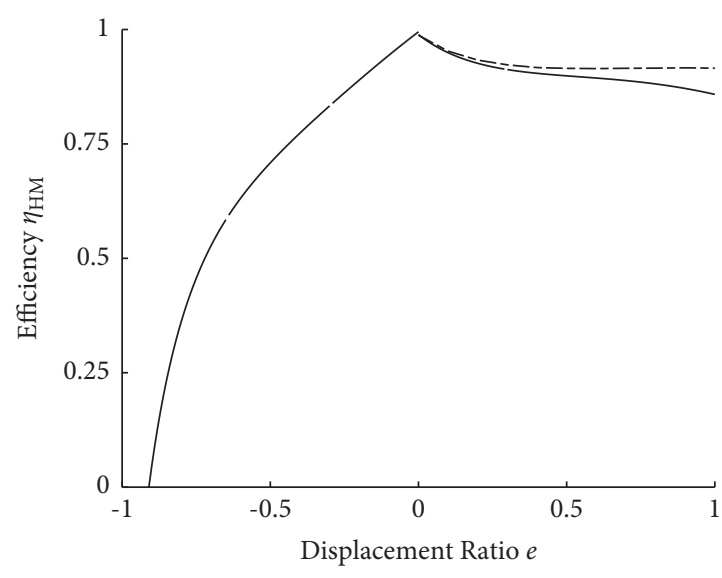

(e)

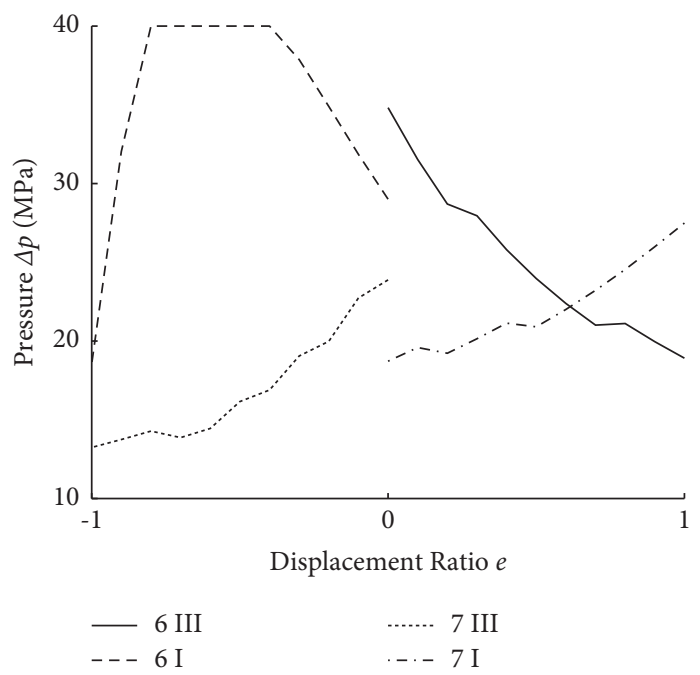

(g)

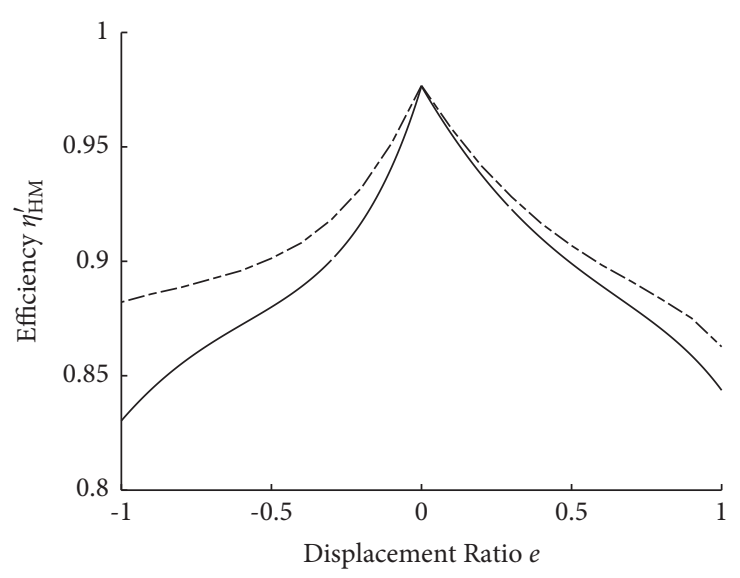

(f)

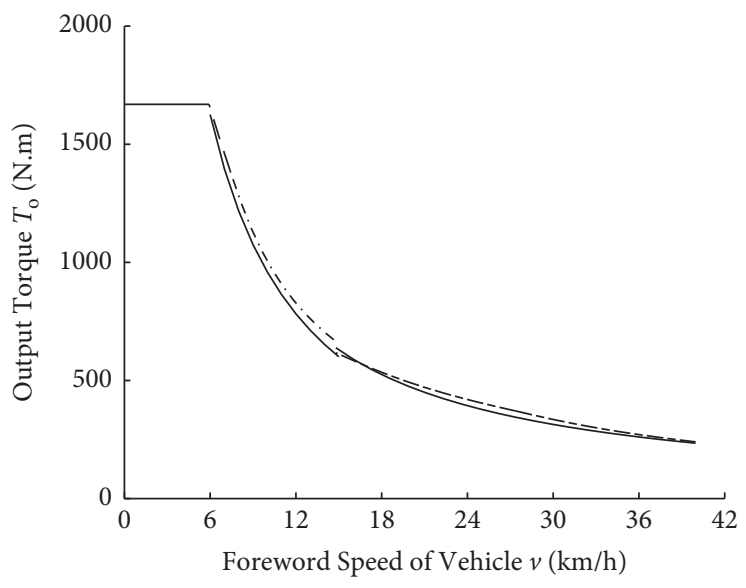

(h)

FIGURE 6: Results of Sts2 with double-variable hydraulic units. (a) In type III with output link 6. (b) In type I with output link 6. (c) In type III with output link 7. (d) In type I with output link 7. (e) Comparison of efficiency with 6. (f) Comparison of efficiency with 7. (g) Curves of $\Delta_{p}$ relative to $e$. (h) Comparison of $T_{o}$ relative to $v$.

of mechanical efficiency $\eta_{m h}$ and volumetric efficiency $\eta_{v}$ cannot be obtained at the same time, and because the positions of $\eta_{v}$ and $\eta_{m h}$ in the efficiency expression are different. As shown by the curves of $\Delta_{p}$ relative to $e$, when $e=0$, the mutations of working pressure are mainly due to the mutations of $e^{\prime}$.

At the start of the operation of the entire machine in type I power flow with output shaft 6 , an effective way to improve system efficiency is to increase the q'vmax of hydraulic unit 2 , as shown in Figure 7, by using the same curved surfaces of $\eta_{v}$ and $\eta_{m h}$ without consideration of the influence of maximum displacement $q_{v \max }^{\prime}$ on system efficiency $\eta_{v}$ and $\eta_{m h}$.

To increase the maximum speed of the vehicle with dual variable units, a maximum speed greater than $60 \mathrm{kph}$ can be obtained by setting variable $e<-2.6$ in the type III power flow with output shaft 7 . Let $e^{\prime \prime}=q_{v} / q_{v \max }$, where $q_{v \text { max }}$ is the maximum value of variable $q_{v}$. To obtain the curved surface that represents $\eta$ relative to $e$ and $e^{\prime \prime}$, the processes of Sts 2 are repeated, with $e^{\prime \prime}\left(=e e^{\prime}\right)$ replacing $e^{\prime}$. The points $\left(e, e^{\prime \prime}, \eta\right)$ that have the highest efficiency for each $e$ are connected.

When $e<-1.8, e^{\prime \prime}=1$, as shown in Figure 8. This condition indicates that the transmission efficiency of HMCVT can be improved when the value of qvmax is increased and $e<-1.8$, but the improvement is not significant.

\section{Test Verification}

The effectiveness of the HMCVT transmission efficiency calculation method proposed in this study is verified, and an HMCVT gearbox with a multisegment univariate unit similar to that in Figure 1 is selected as the test object. The test site of the physical prototype TA1-02 is shown in Figure 9. Torque and speed sensors HBM3000 and HBM5000 with ranges of 3000 and $5000 \mathrm{~N} \mathrm{~m}$, respectively, are installed on the output shaft of the DC variable frequency motor, the output shaft of the HMCVT, and the hydraulic motor of the energy recovery device. System pressure test 


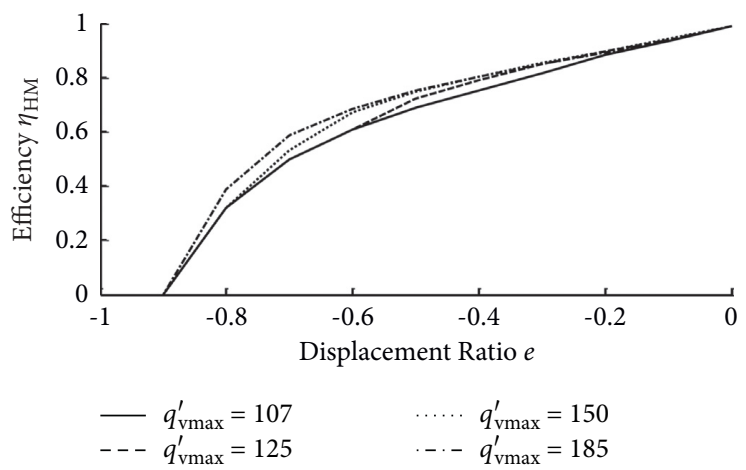

FIGURE 7: Relationship of $\eta_{H M}$ with $e$ and $q_{v \max }^{\prime}$ in type I power flow with output shaft 6.

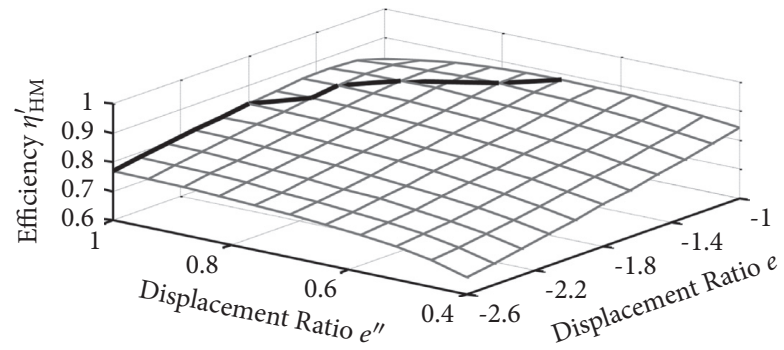

Figure 8: Curved surfaces of $\eta_{H M}^{\prime}$ relative to $e$ and $e^{\prime \prime}$ in type III power flow with output shaft 7 when $e<-1$.

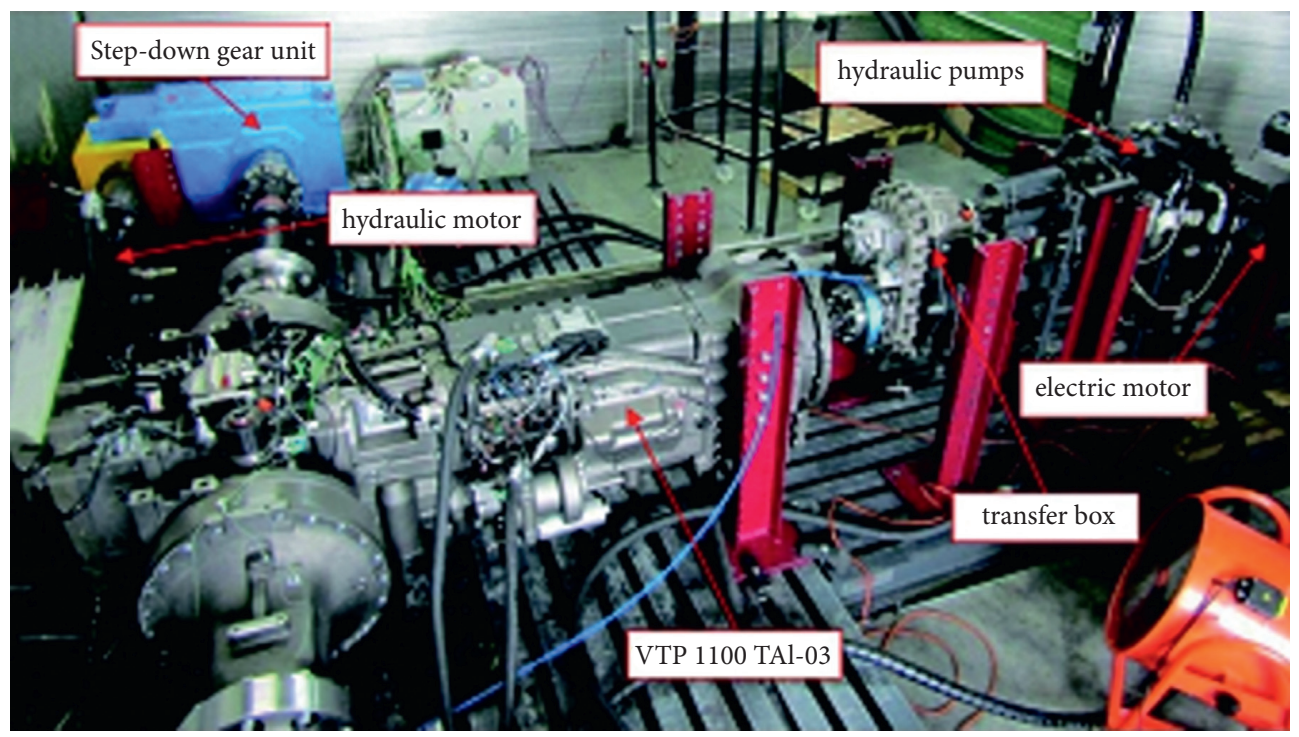

FIGURE 9: Graph of systematic experiments.

data from the transmission TCU are read via CAN bus. The test arrangement is applied in accordance with the gear shift strategy of the tractor's traction conditions. The input torque is stable at $1030 \mathrm{Nm}$, and the input speed is stable at $1700 \mathrm{r} \cdot \mathrm{min}^{-1}$. These values are gradually increased according to the transmission ratio, and A, B, C, D, E, and F are selected for a total of six working conditions for the bench test.
Some of the data collected on site are shown in Figure 10. The input torque is represented by a blue line, the pressure of the $\mathrm{B}$ channel between the hydraulic units is represented by the red line, and the high-pressure-side system pressure is represented by a yellow line.

The transmission efficiency comparison is shown in Figure 11, and the system pressure comparison is shown 


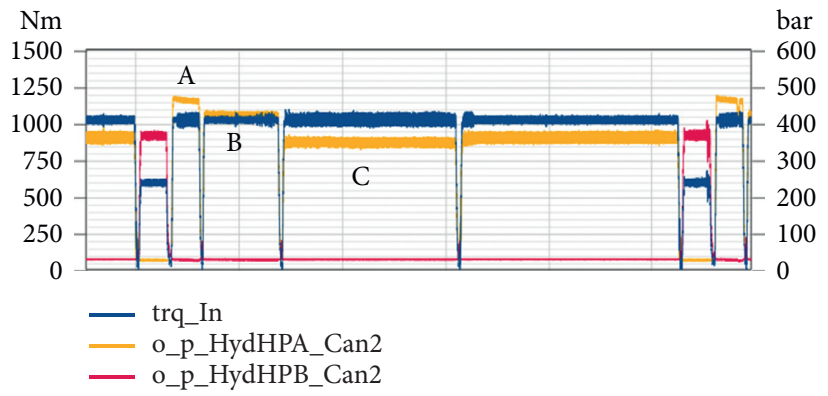

(a)

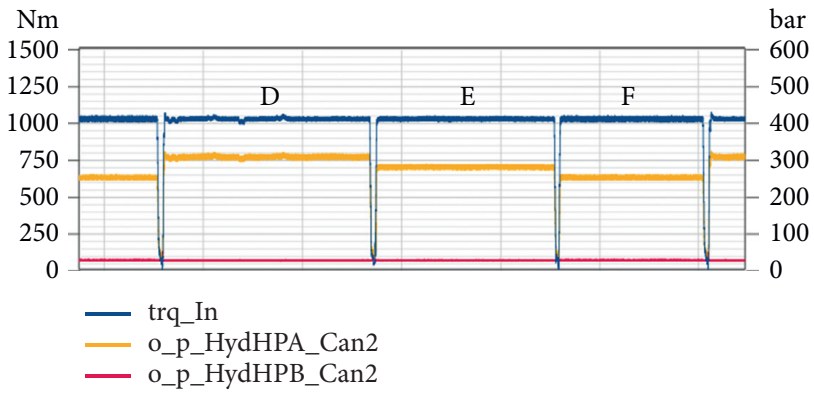

(b)

Figure 10: Measurement test program.

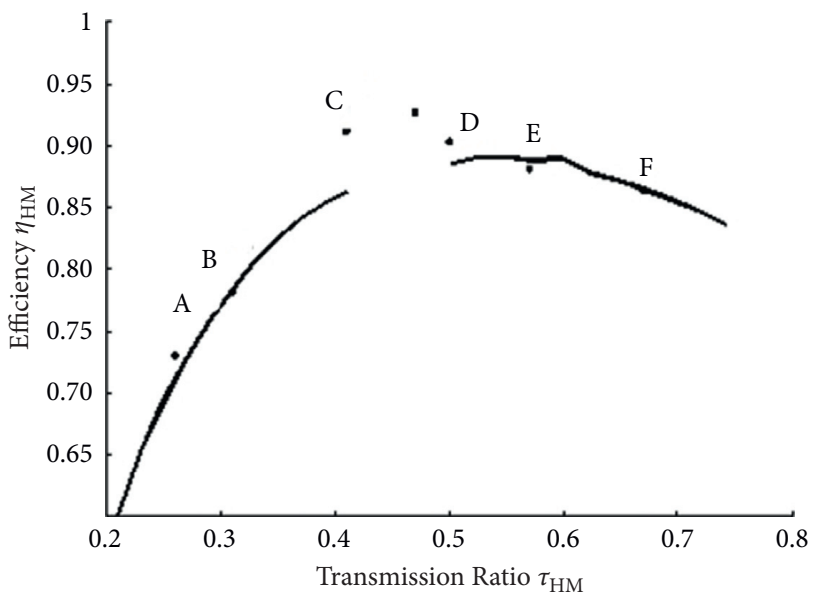

Figure 11: Data on system efficiency.

in Figure 12. In both figures, the star point denotes the test data of the bench under six working conditions, and the solid line is the numerical calculation result of the 22 point positions. The maximum point of the simulation efficiency error is at point $\mathrm{C}$ of the minimum displacement ratio of the type I power flow, and the error is $4.7 \%$. The error for the other operating conditions is less than $2 \%$. Working conditions $\mathrm{C}$ and $\mathrm{D}$ are at the minimum displacement ratio, and the torque of hydraulic units 1 and 2 is small. The efficiency simulation error is large due to the relatively large simulation torque control error and the relatively large influence of the nonlinear fitting effect of the HST hydraulic system. The efficiency simulation values of the operating conditions, except for operating conditions A, $\mathrm{C}$, and $\mathrm{D}$, are greater than the actual measured values, because the simulation does not consider transmission churning loss and bearing loss. In Figure 12, the simulated pressure curve effectively reflects the working characteristics of the hydraulic system. As the transmission ratio increases, the HST system pressure gradually decreases with a hyperbolic curve, and the system works in type I and III power flow states. The high-pressure side of the system shows no changes. The simulated pressure curve is lower than the actual measured value, and the transmission power of the HST system is lower than that in the

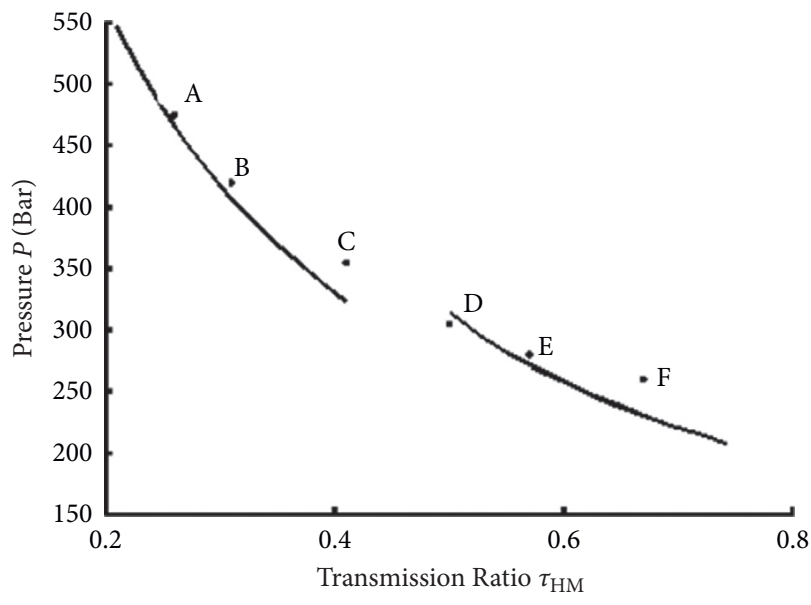

FIgURE 12: Data on system pressure.

actual working conditions, which is the main reason for the high efficiency of the simulation.

\section{Conclusions}

Two parameters, namely, hydraulic transmission asymmetry $\lambda$ and maximum angular velocity ratio $\tau_{H M}^{\max }$, are introduced to express the structural characteristics of hydromechanical continuously variable transmission (HMCVT) with a variable speed range of 0 to the maximum. This approach provides a means to solve the classification problem of HMCVT.

The relationship between mechanical system transmission and the parameters of the hydraulic system is established by the mechanical system demanding surface. The theory of meshing power is applied to determine the torque ratio coefficient $\Delta_{i}$ of an epicyclic gear train (EGT) with two DOFs. The expression of HMCVT overall efficiency is simplified and unified by torque ratio coefficient $\Delta_{i}$ in the steady state under the rated conditions of the diesel engine.

The control theory of parameters $e^{\prime}$ and $\mathrm{e}^{\prime \prime}$ is revealed to obtain the maximum transmission efficiency of HMCVT. The application method of this theory is introduced through numerical examples, so that hydraulic system components can be selected properly, and the output torque can be predicted. 
The physical prototype bench test conducted using TA102 multisection univariate-unit HMCVT and the simulation show that the maximum efficiency simulation error is at point $\mathrm{C}$ of the minimum displacement ratio of the type $\mathrm{I}$ power flow, and the error is $4.7 \%$. The error for the other operating conditions is less than $2 \%$. The simulated pressure curve effectively reflects the working characteristics of the hydraulic system. As the transmission ratio increases, the HST system pressure gradually decreases in a hyperbolic curve, which verifies the effectiveness of the HMCVT efficiency analysis steps.

For the study of transmission efficiency of future transmissions, the influence of diesel engine working characteristics, overall slip rate, and changes in traction force on transmission efficiency will be comprehensively considered.

\section{Data Availability}

The data described in this study could be fully provided by the authors.

\section{Conflicts of Interest}

The authors declare that there are no conflicts of interest regarding the publication of this study.

\section{Acknowledgments}

This work was supported by the Jiangsu International Science and Technology Cooperation Project (BZ2020061), Jiangsu Provincial Agricultural Science and Technology Independent Innovation Fund (CX (19)3071), and Development Program of Jiangsu Province (BE2019337).

\section{References}

[1] J. H. Kress, "Hydrostatic power-splitting transmissions for wheeled vehicles- classification and theory of operation," SAE International Journal of Engines, vol. 77, pp. 2282-2306, 1968.

[2] J. Wang, C. Xia, X. Fan, and J. Cai, "Research on transmission characteristics of hydromechanical continuously variable transmission of tractor," Mathematical Problems in Engineering, vol. 2020, Article ID 6978329, 14 pages, 2020.

[3] L. Xu, Z. Zhou, M. Zhang, and F. Cao, "Speed ratio matching strategies of hydro-mechanical continuously variable transmission system of tractor," Journal of China Agricultural University, vol. 2, no. 4, pp. 94-98, 2006.

[4] H. T. Xue, D. Y. Ding, Z. M. Zhang, M. Wu, and H. Q. Wang, "A fuzzy system of operation safety assessment using multimodel linkage and multi-stage collaboration for in-wheel motor," IEEE Transactions on Fuzzy Systems, 2021.

[5] K. T. Renius, "Trends in tractor design with particular reference to europe," Journal of Agricultural Engineering Research, vol. 57, no. 1, pp. 3-22, 2013.

[6] H. T Xue, M. Wu, Z. M. Zhang, and H. Q. Wang, "Intelligent diagnosis of mechanical faults of in-wheel motor based on improved artificial hydrocarbon networks," ISA Transaction, 2021.

[7] M. Xiao, J. Zhao, Y. Wang, F. Yang, J. Kang, and H. Zhang, "Research on system identification based on hydraulic pumpmotor of HMCVT," Engineering in Agriculture, Environment and Food, vol. 12, no. 4, pp. 420-426, 2019.
[8] W. Wei, R. Cong, T. Xue, A. D. Abraham, and C. Yang, "Surface roughness and chip morphology of wood-plastic composites manufactured via high-speed milling," Bioresources, vol. 16, no. 3, pp. 5733-5745, 2021.

[9] G. M. Wang, S. H. Zhu, S. H. Wang, L. Shi, X. D. Ni, and D. Ouyang, "Speed ratio control of tractor hydraulic mechanical continuously variable transmission," Transactions of the Chinese Society of Agricultural Engineering, vol. 29, no. 7, pp. 17-23, 2013.

[10] Z. Cheng, Z. Lu, and F. Dai, "Research on HMCVT efficiency model based on the improved SA algorithm," Mathematical Problems in Engineering, vol. 2019, Article ID 2856908, 10 pages, 2019.

[11] J. Y. Li, Q. C. Hu, C. F. Zong, and T. J. Zhu, "Power analysis and efficiency calculation of multistage micro-planetary transmission," Energy Procedia, vol. 141, pp. 654-659, 2017.

[12] J. Y. Li and Q. C. Hu, "Power analysis and efficiency calculation of the complex and closed planetary gears transmission," Energy Procedia, vol. 100, pp. 423-433, 2016.

[13] M. Awadallah, P. Tawadros, P. Walker, and N. Zhang, "Dynamic modelling and simulation of a manual transmission based mild hybrid vehicle," Mechanism and Machine Theory, vol. 112, pp. 218-239, 2017.

[14] E. Pennestri and F. Freudenstein, "The mechanical efficiency of epicyclic gear trains," Journal of Mechanical Design, vol. 115, no. 3, pp. 645-651, 1993.

[15] G. Mantriota and E. Pennestrì, "Theoretical and experimental efficiency analysis of multi-degrees-of -freedom epicyclic gear trains," Multibody System Dynamics, vol. 9, no. 4, pp. 389-408, 2003.

[16] J. M. del Castillo, "The analytical expression of the efficiency of planetary gear trains," Mechanism and Machine Theory, vol. 37, no. 2, pp. 197-214, 2002.

[17] F. Yang, J. Feng, and H. Zhang, "Power flow and efficiency analysis of multi-flow planetary gear trains," Mechanism and Machine Theory, vol. 92, pp. 86-99, 2015.

[18] W. H. Wei, Y. L. Li, and Y. T. Li, Y. Li, C. Yang, Research on tool wear factors for milling wood-plastic composites based on response surface methodology," Bioresources, vol. 16, no. 1, pp. 151-162, 2021.

[19] X. M. Xu and P. Lin, "Parameter identification of sound absorption model of porous materials based on modified particle swarm optimization algorithm," PLOS ONE, vol. 16, no. 5, pp. 1-16, 2021.

[20] R. H. Macmillan, "Power flow and loss in differential mechanisms," Journal of Mechanical Engineering Science, vol. 3, no. 1, pp. 37-41, 1961.

[21] C. Chen and T. T. Liang, "Theoretic study of efficiency of twodof of epicyclic gear transmission via virtual power," Journal of Mechanical Design, vol. 133, no. 3, pp. 1-7, 2011.

[22] L. Mangialardi and G. Mantriota, "Power flows and efficiency in infinitely variable transmissions," Mechanism and Machine Theory, vol. 34, no. 7, pp. 973-994, 1999.

[23] G. Mantriota, "Performances of a series infinitely variable transmission with type I power flow," Mechanism and Machine Theory, vol. 37, no. 6, pp. 579-597, 2002.

[24] G. Mantriota, "Performances of a parallel infinitely variable transmissions with a type II power flow," Mechanism and Machine Theory, vol. 37, no. 6, pp. 555-578, 2002.

[25] C. Chen and J. Angeles, "Virtual-power flow and mechanical gear-mesh power losses of epicyclic gear trains," Journal of Mechanical Design, vol. 129, no. 1, pp. 107-113, 2007.

[26] Y. I. Xiao, S. J. Jiao, Z. F. Liu, T. Q. Zhang, and S. G. Long, "Research on key technical parameters of full hydraulic bulldozer," China Journal of Highway and Transport, vol. 17, no. 23, pp. 119-123, 2004. 